\title{
Proteomics analysis of potential serum biomarkers for insulin resistance in patients with polycystic ovary syndrome
}

\author{
LI LI $^{1 *}$, JING ZHANG ${ }^{2 *}$, JING ZENG $^{1}$, BILING LIAO ${ }^{1}$, XIUHONG PENG $^{1}$, TIANTIAN LI ${ }^{1}$, JIEMING LI $^{1}$,

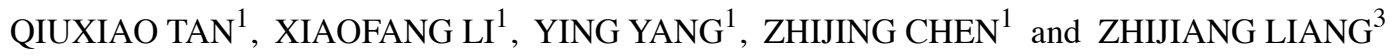

\begin{abstract}
${ }^{1}$ Department of Obstetrics and Gynecology, Guangdong Women and Children Hospital, Guangzhou, Guangdong 510010;
${ }^{3}$ Department of Public Health, Guangdong Women and Children Hospital, Guangzhou, Guangdong 510010, P.R. China
\end{abstract} \\ ${ }^{2}$ Department of Gynecology, Family Planning Research Institute of Guangdong Province, Guangzhou, Guangdong 510600;
}

Received March 13, 2019; Accepted February 11, 2020

DOI: $10.3892 /$ ijmm.2020.4522

\begin{abstract}
The aim of the present study was to identify potential serum biomarkers for insulin resistance (IR) in patients with polycystic ovary syndrome (PCOS) by comparing the differences in serum protein expression levels between PCOS patients with and without IR. PCOS patients aged from 18 to 35 years were recruited at Guangdong Women and Children's Hospital from January, 2013 to February, 2014. A total of 218 PCOS patients were enrolled and divided into the insulin resistance (PCOS-IR) and non-insulin resistance (PCOS-NIR) groups according to their homeostasis model assessment of insulin resistance. Two-dimensional difference gel electrophoresis (2D-DIGE) and matrix-assisted laser desorption/ionization time-of-flight mass spectrometry (MALDI-TOF-MS/MS) techniques were used to identify differences in protein expression levels between the PCOS-IR and PCOS-NIR groups. The present study demonstrated that the total cholesterol $(\mathrm{TCH})$, triglycerides (TG), low-density lipoprotein (LDL), fasting plasma glucose (FPG), 3-h blood glucose (3hBG) and uric acid (UA) levels in the PCOS-IR group were higher than those in the PCOS-NIR group $(\mathrm{P}<0.05)$. Between the
\end{abstract}

Correspondence to: Dr Zhijiang Liang, Department of Public Health, Guangdong Women and Children Hospital, 3 Guang Yuan Xi Lu, Yuexiu, Guangzhou, Guangdong 510010, P.R. China

E-mail: liangzhijiang07@163.com

${ }^{*}$ Contributed equally

Abbreviations: PCOS, polycystic ovary syndrome; IR, insulin resistance; BW, body weight; BMI, body mass index; TT, total testosterone; FAI, free androgen index; IAUC, insulin area under curve; HOMA-IR, homeostasis model assessment of insulin resistance; SHBG, sex hormone binding globulin; TG, triglycerides; LDL, low-density lipoprotein; FPG, fasting plasma glucose; UA, uric acid; HDL-C, high-density lipoprotein; APOC3, apolipoprotein C3

Key words: polycystic ovary syndrome, proteomics, insulin resistance, apolipoprotein $\mathrm{C} 3$
PCOS-IR and PCOS-NIR groups, a total of 20 differentially expressed protein spots were detected by 2D-DIGE. Among these, 4 proteins, namely afamin, serotransferrin, complement C3 and apolipoprotein C3 (APOC3), were also identified by MALDI-TOF-MS/MS. The alteration of APOC3 was further confirmed by western blot analysis and enzyme-linked immunosorbent assay (ELISA). The present study also confirmed that the expression level of APOC3 was positively associated with the homeostasis model assessment of insulin resistance (HOMA-IR). On the whole, the data indicate that APOC3 may be a potential diagnostic marker for PCOS-IR patients.

\section{Introduction}

Polycystic ovary syndrome (PCOS) is the most common hormonal disorder affecting women between the ages of 18 and 44 years. The characteristics of PCOS include hyperandrogenism, ovulatory dysfunction and polycystic ovaries (1). The pathogenesis of PCOS remains unclear and is generally considered to be caused by a combination of genetic and environmental factors, such as long-term exposure to high levels of androgens in utero. The prevalence of PCOS depends on the selection of the diagnostic criteria. Epidemiological studies based on the Rotterdam criteria revealed that approximately $18 \%$ of women suffer from PCOS, which affects 116 million women worldwide $(2,3)$. At present, PCOS is incurable. Current treatments, such as the administration of metformin and anti-androgen to improve hyperandrogenism, the birth control pill to regulate menstruation, lifestyle changes, such as weight loss, exercise, etc., all address mainly the symptoms of PCOS (4). The United States spent a reported $\$ 4.36$ billion on medical care for its 4 million patients with PCOS in 2005 (5). PCOS is one of the leading causes of infertility today, severely affecting the health of women.

Insulin resistance (IR) refers to the abnormal physiological phenomena including the weakening effects of pancreatic $\beta$ cells and the reduced sensitivity of peripheral tissue to insulin. In 1980, Burghen et al first proposed the involvement of IR in PCOS, and in 1989 Dunaif et al found that approximately $20 \%$ of obese women with PCOS suffered from IR $(6,7)$. A previous study demonstrated that a number of lean patients with PCOS also suffered from IR, and the lean patients with PCOS with normal insulin levels were significantly more likely 
to suffer from post-prandial hyperinsulinemia (8). According to a systematic review and meta-analysis, and as previously demonstrated, patients with PCOS and IR (PCOS-IR) were more likely to have long-term complications, such as glucose metabolic abnormalities, type 2 diabetes, cardiovascular disease and unopposed estrogen effects on the endometrium, compared with patients with PCOS alone $(9,10)$, thus indicating that IR and hyperinsulinism may play an important role in the pathophysiology of PCOS. A previous study also confirmed that women with PCOS and IR were more inclined to suffer from metabolic disorders, such as upregulated blood glucose, blood lipid and uric acid levels (11).

It has been demonstrated that early diagnosis and timely treatment can significantly delay the occurrence of short-term and long-term severe complications, such as infertility, type 2 diabetes and endometrial carcinoma in patients with PCOS $(12,13)$. For instance, a number of scholars used Diane-35 and metformin for the treatment of PCOS-IR women diagnosed with early endometrial cancer. Following 6 months of co-treatment, body weight (BW), body mass index (BMI), total testosterone (TT), free androgen index (FAI), insulin area under curve (IAUC) and homeostasis model assessment of insulin resistance (HOMA-IR) markers were significantly reduced, in combination with a significant increase in sex hormone binding globulin (SHBG). Diane-35 and metformin co-treatment successfully transformed the hyperplasia of endometrial into normal endometrial and reversed the progression of endometrial carcinoma (14).

Currently, various methods are available for the to diagnosis of IR, among which the hyperinsulinemic euglycemic clamp (HEC) is the golden standard $(15,16)$. However, due to the high cost and complications associated with the surgery, its use is limited in clinical practice. It has been demonstrated that fasting insulin, an assessment index for IR, can only be applied to the non-diabetic group (17). HOMA-IR has a good association with HEC, which is suitable for both diabetics and non-diabetics. However, the value of HOMA-IR is calculated based on the fasting homeostasis data, which cannot truly reflect the dynamic process of insulin in the body $(18,19)$. Therefore, it is important to identify specific protein markers which are sensitive to distinguishing the IR status of PCOS patients. The present study conducted a proteomics-based approach to identify and select novel protein markers associated with IR in the serum of patients with PCOS-IR.

\section{Materials and methods}

Clinical specimens. Total 218 patients with PCOS were recruited at Guangdong Maternal and Child Health Hospital from January, 2013 to February, 2014. The patients who were recruited for the study had to simultaneously meet the following four criteria: i) Subjects were $18-35$ years of age; ii) the criteria for the diagnosis of PCOS were based on the revised diagnostic criteria announced in the 2003 by the European Society for Human Reproduction and Embryology/American Society for Reproductive Medicine (ESHRE/ASRM), which includes two of the following: Clinical and/or biochemical hyperandrogenism; oligo-ovulation or anovulation; polycystic ovaries detected by ultrasound; iii) the subjects had no medication history over the past 3 months prior to the first diagnosis that confirmed PCOS; iv) subjects voluntary participated and conformed well to this clinical study. The exclusion criteria were the following: i) Hormone drugs or drugs that affect insulin production were taken during the past 3 months prior to enrollment; ii) pregnant or lactating women; iii) patients with cardiovascular disease, liver and kidney, hematopoietic system and other diseases; iv) patients suspected to suffer from malignant tumors and adrenal disease; v) patients with glucose-6-phosphate deoxydase deficiency; and vi) BMI $<18 \mathrm{~kg} / \mathrm{m}^{2}$. The patients were assessed according to the homeostasis model assessment and were divided into the PCOS-IR group $(n=84)$ and the PCOS-NIR group $(n=134)$. Blood specimens were obtained at the early stage of the follicular phase (3-5 days of the cycle) in women with regular menstruation and randomized in women with amenorrhea. The present study was approved by the Ethics Committee of the Guangdong Provincial Maternal and Child Health Hospital and all patients signed written informed consent to participate.

Sample preparation. Following the collection, all samples were placed at room temperature for $2 \mathrm{~h}$ and the supernatants were then centrifuged at $15,000 \times \mathrm{x}$ and at $4^{\circ} \mathrm{C}$ to remove lipids. Albumin and $\operatorname{IgG}$ were removed using the Proteo Extract Albumin/IgG Removal kit (Merck \& Co., Inc.) according to the manufacturer's instructions. Subsequently, the samples were resuspended in lysis buffer $(30 \mathrm{mM}$ Tris- $\mathrm{HCl}, 7 \mathrm{M}$ urea, $2 \mathrm{M}$ thiourea, $4 \%$ CHAPS, at $\mathrm{pH} 8.5$ ), and incubated on ice for $30 \mathrm{~min}$. The suspended samples were then centrifuged at $15,000 \times \mathrm{g}$ and at $4^{\circ} \mathrm{C}$ for $30 \mathrm{~min}$. Protein concentrations were determined using the 2D Quant kit (GE Healthcare BioSciences) according to the manufacturer's protocol. Finally, the proteins were freeze-dried. All the other reagents were supplied by Sigma-Aldrich; Merck KGaA unless otherwise indicated.

Two-dimensional difference gel electrophoresis (2D-DIGE). Serum from patients in the PCOS-IR group and the PCOS-NIR group was randomly selected for 2D-DIGE analysis. Due to financial constraints, 20 subjects out of the total nmber of clinical samples were randomly selected to perform 2D-DIGE analysis. 2D-DIGE is the most commonly used method in proteomics. 2D-DIGE combined with digital image analysis markedly improves the statistical evaluation of proteome variation $(20,21)$. The amount of $50 \mu \mathrm{g}$ of proteins was minimally labelled with CyDyes at the ratio of $1 \mu \mathrm{g}$ protein: $8 \mathrm{pmol}$ $\mathrm{Cy} 3$ or Cy5 protein-labeling dye (GE Healthcare BioSciences) according to the manufacturer's protocol. $\mathrm{Cy} 3$ or $\mathrm{Cy} 5$ were used to label the samples and Cy2 was used to label the internal standard (a pool of all the samples). Each labeled sample was applied to a $24-\mathrm{cm}$ immobilized $\mathrm{pH}$ gradient gel strip (immobilized $\mathrm{pH}$ gradient strip $\mathrm{pH} 3$ to $10 \mathrm{NL}$ ) for separation in the first dimension. The first dimension isoelectric focusing was carried out at $20^{\circ} \mathrm{C}$ in IPG phor III (GE Healthcare BioSciences). The strips were then loaded onto a $24 \times 24 \mathrm{~cm} 12 \%$ polyacrylamide gel using low fluorescence glass plates and subjected to an electric field in the DALT Six (GE Healthcare BioSciences). Subsequently, the gels were scanned on a Typhoon 9400 imager (GE Healthcare BioSciences) and analyzed with the DeCyder 2D Software V6.5 (GE Healthcare BioSciences). The protein spots, which were shown to be differentially expressed between both groups (filtering conditions: At least $50 \%$ change 
Table I. Basic clinical data of the patients in the PCOS-IR and the PCOS-NIR groups.

\begin{tabular}{|c|c|c|c|c|c|c|}
\hline Group & No. of patients & Age (years) & BMI $\left(\mathrm{kg} / \mathrm{cm}^{2}\right)$ & $\mathrm{WC}(\mathrm{cm})$ & $\mathrm{HC}(\mathrm{cm})$ & WHR \\
\hline PCOS-NIR & 134 & $24.4 \pm 4.6$ & $21.02 \pm 3.06$ & $74.65 \pm 7.99$ & $96.97 \pm 82.83$ & $0.83 \pm 0.09$ \\
\hline PCOS-IR & 84 & $25.2 \pm 5.9$ & $25.78 \pm 3.65$ & $87.3 \pm 12.67$ & $98.74 \pm 7.13$ & $0.88 \pm 0.11$ \\
\hline $\mathrm{t}$ value & & -1.098 & -10.14 & -8.54 & -0.183 & -3.844 \\
\hline P-value & & 0.274 & $<0.001^{\mathrm{a}}$ & $<0.001^{\mathrm{a}}$ & 0.855 & $<0.001^{\mathrm{a}}$ \\
\hline
\end{tabular}

All statistical analyses were performed using SPSS 20.0 software. The data were analyzed using an independent-samples t-test and are

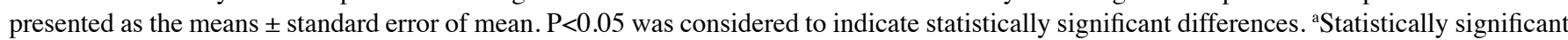
differences between the PCOS-NIR and PCOS-IR groups. PCOS-IR, polycystic ovary syndrome patients complicated by insulin resistance; PCOS-NIR, polycystic ovary syndrome patients without insulin resistance; BMI, body mass index; WC, waistline circumference; HC, hip circumference; WHR, waist-hip ratio.

of ratios between both groups. The spot picking gel without labeling by CyDyes was made with $600 \mu \mathrm{g}$ of pooled protein sample and stained with colloidal coomassie blue G-250. The matched spots were selected by the Ettan Spot Picker (GE Healthcare BioSciences).

Matrix-assisted laser desorption ionization/time off light MS (MALDI-TOF-MS/MS) analysis and proteinidentification. The collected spots were destained with $50 \%$ acetonitrile/100 mM $\mathrm{NH}_{4} \mathrm{HCO}_{3}$. After $10 \mathrm{~min}, 2 \mu \mathrm{l}$ of $25 \mathrm{ng} / \mathrm{ml}$ trypsin diluted in $50 \mathrm{mM} \mathrm{NH} \mathrm{HCO}_{3}$ were added to each gel piece and $30 \mu \mathrm{l}$ of $50 \mathrm{mM} \mathrm{NH}_{4} \mathrm{HCO}_{3}$ were then added followed by incubation overnight at $37^{\circ} \mathrm{C}$. The peptide mixtures from the gel pieces were extracted and dry-digested using a vacuum pump. Subsequently, $2 \mu 150 \%$ acetonitrile/0.1\% TFA and $0.5 \mu 1$ matrix solution containing CHCA saturated in 50\% acetonitrile $/ 0.1 \%$ TFA were used to redissolve the powder. The samples were then analyzed using the ABI 4800 Proteomics Analyzer MALDI-TOF/TOF mass spectrometer (Applied Biosystems). For most mass spectrometers, the upper limit for $\mathrm{m} / \mathrm{z}$ is between 650 and 800 . MS/MS analyses were performed at collision energy of $2 \mathrm{KV}$ with air. The Mascot search engine (version 2.1, Matrix Science) and the GPS Explorer ${ }^{\mathrm{TM}}$ software version 3.6.2 (Applied Biosystems) were used to explore the tandem mass spectra and peptide and protein. The Mascot searching engine was used to identify the protein.

Western blot analysis. Proteins used for western blot analysis were extracted from human serum by ultracentrifugation at $15,000 \mathrm{x} \mathrm{g}$ for $30 \mathrm{~min}$ at $4^{\circ} \mathrm{C}$. The protein concentrations were then quantified by the bicinchoninic acid (BCA) protein determination method and a total of $100 \mu \mathrm{g}$ proteins were selected for further analysis. Firstly, 2 volumes of acetonitrile were used to remove the peak proteins in the serum. The remaining proteins were divided into equal portions, one for detecting the target protein, and the other for Coomassie bright blue staining. The proteins were separated by $12 \%$ polyacrylamide gel electrophoresis and the proteins were then transferred onto a nitrocellulose membrane. The membrane was then incubated in blocking buffer for $1 \mathrm{~h}$ at room temperature and incubated with the primary anti-apolipoprotein $\mathrm{C} 3$ (APOC3) rabbit monoclonal antibody $\left(1: 2,000 ;\right.$ ab76305, Abcam) overnight at $4^{\circ} \mathrm{C}$, followed by incubation with a horseradish peroxidase-conjugated secondary antibody (1:5,000; 7074, Cell Signaling Technology) for $1 \mathrm{~h}$ at room temperature. The signal was visualized by ECL solution and the ImageQuant image analysis system (optical storm scanners, Molecular Dynamics).

Enzyme-linked immunosorbent assay (ELISA). Serum APOC3 concentrations were measured using the Human APOC3 ELISA kit (Blue Gene Biotech) according to the manufacturer's protocols. A solution was added to terminate the reaction, which turned the solution yellow. The optical density (OD) of plasma APOC3 was measured spectrophotometrically at $450 \mathrm{~nm}$ using a microplate reader (PW-812, Shenzhen Huisong Technology Development Co., Ltd.). A standard curve was plotted according to OD of the concentration of standards. The APOC3 concentration in each sample was examined from this standard curve.

Statistical analysis. For statistical analysis, SPSS 20.0 software was used. In order to prevent and control the result error caused by the quantitative difference between the groups, the same number of cases, which were used in the subsequent proteomics analysis, western blot analysis and so on, were selected for comparison. The parametric variables were analyzed by normal distribution and homogeneity of variance. The Student's t-test was applied for comparisons between the PCOS-IR group and the PCOS-NIR group. For non-normally distributed data, the Wilcoxon rank sum test was used. The area under curve (AUC) value, optimal cut-off value, sensitivity and specificity were determined using receiver-operating characteristic (ROC) curve. Linear regression analysis was performed to examine the correlation between APOC 3 and HOMA-IR. P<0.05 was considered to indicate statistically significant differences. The data are presented as the means \pm standard error of mean (SEM).

\section{Results}

Severe disruption of metabolic parameters in the PCOS-IR group. A total of 218 PCOS patients were recruited at Guangdong Women and Children's Hospital from January, 2013 to February, 2014. Among these, 84 patients with HOMA-IR $\geq 2.69$ were recruited into the PCOS-IR group and 134 patients with HOMA-IR <2.69 were recruited into the PCOS-NIR group. As illustrated in Table I, statistically 


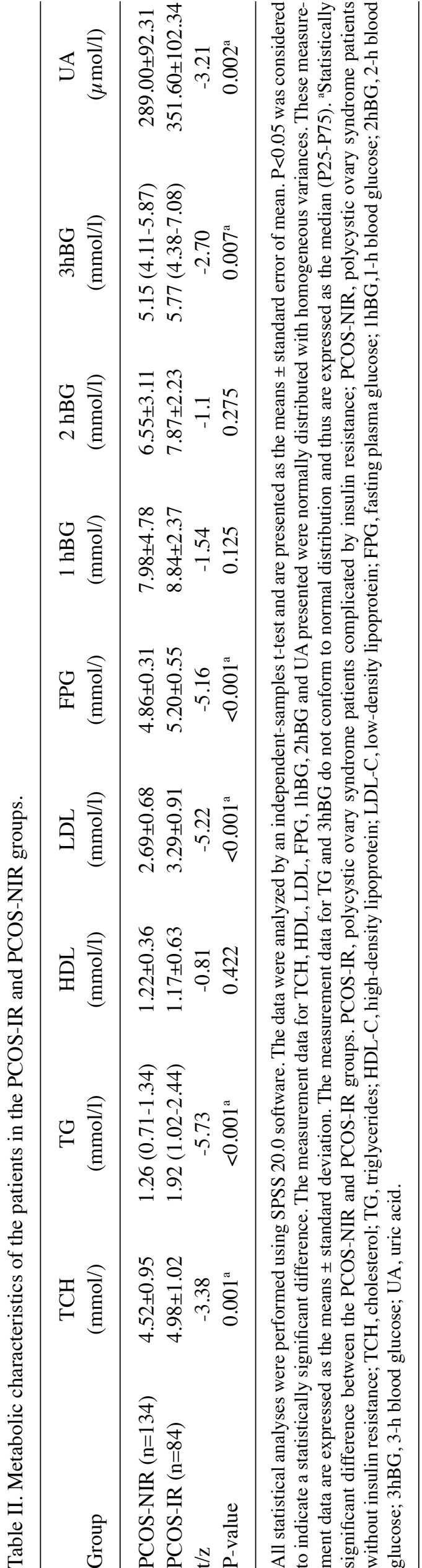

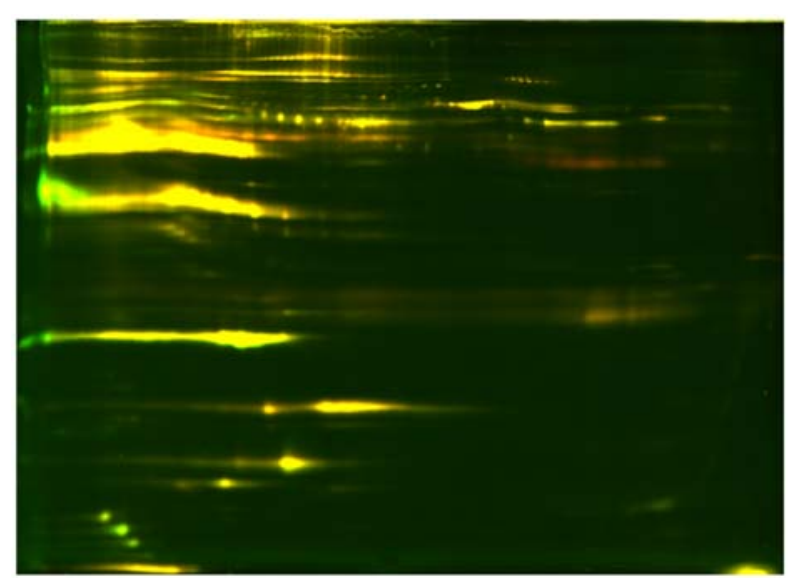

Figure 1. Overlapped sections of the two-dimensional fluorescence difference in gel electrophoresis (2D-DIGE) proteome map of PCOS-IR group and PCOS-NIR group. Cy2, Cy3 and Cy5 images appear as yellow, green and red using Image Quant TL software. Green spots indicate upregulated proteins in the PCOS-IR group, while orange indicates downregulated proteins in the PCOS-IR group. Yellow spots indicate the proteins which exhibited no difference between both groups. 2D-DIGE, two-dimensional difference gel electrophoresis; PCOS, polycystic ovary syndrome; IR, insulin resistance.

significantly differences were observed in BMI, waist circumference (WC) and the waist-hip ratio (WHR) between the PCOS-IR and the PCOS-NIR groups $(\mathrm{P}<0.001)$. The biochemical results revealed that triglycerides (TG), cholesterol $(\mathrm{TCH})$, low-density lipoprotein (LDL), fasting plasma glucose (FPG) and uric acid (UA) levels were significantly higher in the PCOS-IR group $(\mathrm{P}<0.01)$ than in the PCOS-NIR group. The level of 3-h blood glucose ( $3 \mathrm{hBG})(\mathrm{Z}=-2.70, \mathrm{P}=0.007)$ was also higher in the PCOS-IR group. No significant differences were observed in the levels of high-density lipoprotein (HDL), 1-h blood glucose (1hBG) and 2-h blood glucose (2hBG) between the groups $(\mathrm{P}>0.05)$ (Table II).

Significantly differences in proteomics results between the PCOS-IR and PCOS-NIR groups. A total of 12 paired serum samples from the PCOS-IR and PCOS-NIR groups were randomly selected for 2D-DIGE and the MALDI-TOF-MS/MS analysis. A section of the gel labeled with the DIGE dyes is presented in Fig. 1. Based on the difference of an at least $50 \%$ ratio change between the PCOS-IR and PCOS-NIR group, 20 spots were recognized and marked in Fig. 2. Among the 20 different proteins, only 4 proteins were identified by MALDI-TOF-MS/MS, namely afamin (871), serotransferrin (975), complement C3 $(1,028)$ and APOC3 $(1,955$ and 2,012), and these are marked by red rectangles in Fig. 2.

Protein expression level of APOC3 in the PCOS-IR group is higher than that in the PCOS-NIR group. To further verify the differential protein expression between the PCOS-IR and PCOS-NIR groups, western blot analysis was performed. As illustrated in Fig. 3, the APOC3 expression level was higher in the PCOS-IR group compared with that in the PCOS-NIR group. To further investigate the diagnostic value of serum APOC 3 in patients with PCOS, ELISA was performed to detect the APOC3 levels in 80 PCOS-IR and 80 PCOS-NIR samples. The area under the receiver operator characteristic curve was 0.936 (95\% CI, 0.901-0.972); the Youden index was 


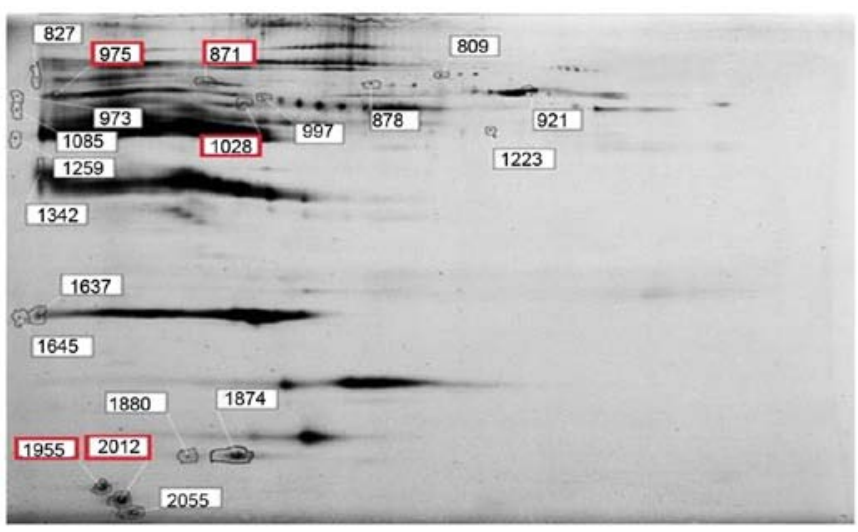

Figure 2. A total of 20 differentially expressed protein spots in the PCOS-IR group and the PCOS-NIR group analyzed using DeCyder 2D software. The image was obtained with a Typhoon 9400 scanner at $633 / 670 \mathrm{~nm}$ excitation/emission wavelengths. Among the 20 different proteins, only 4 proteins were identified by MALDI-TOF-MS/MS, which were afamin (871), serotransferrin (975), complement C3 (1028) and APOC3 (1955 and 2012) and these are marked by red rectangles. PCOS, polycystic ovary syndrome; IR, insulin resistance.

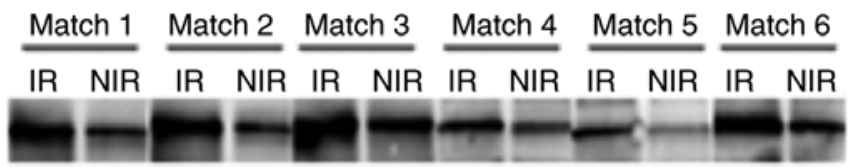

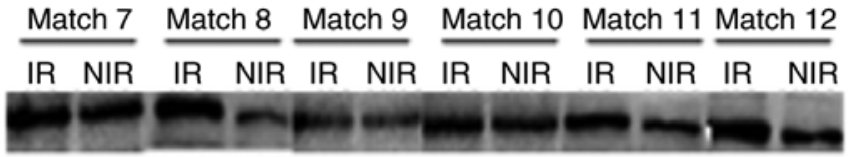

Figure 3. Serum APOC3 protein expression in patients in the PCOS-IR and PCOS-NIR groups examined by western blot analysis. PCOS, polycystic ovary syndrome; IR, insulin resistance. APOC3, apolipoprotein C3.

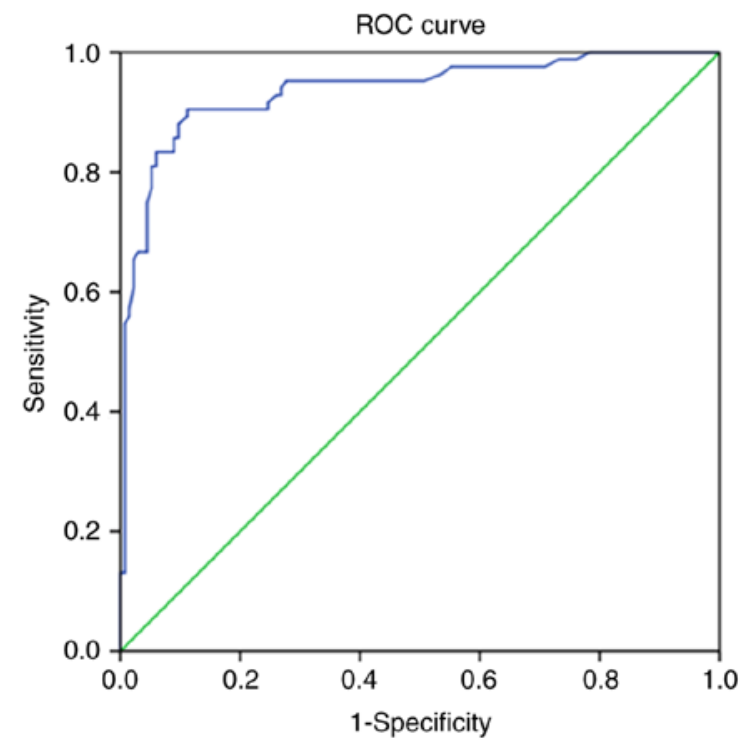

Figure 4. Receiver operator characteristic (ROC) curve based on 80 paired ELISA results for APOC 3 in discriminating PCOS-IR from PCOS-NIR The area under the ROC curve was measured at 0.936 (95\% CI, 0.901-0.972); the cut-off value of ApoC 3 was measured at $10.42 \mathrm{ng} / \mathrm{ml}$; the sensitivity was measured at $88.81 \%$, and the specificity was measured at $90.48 \%$. PCOS polycystic ovary syndrome; IR, insulin resistance. APOC3, apolipoprotein $\mathrm{C} 3$. largest when the demarcation value was $10.42 \mathrm{ng} / \mathrm{ml}$, the sensitivity was $88.81 \%$, and the specificity was $90.48 \%$ (Fig. 4).

Serum APOC3 levels in patients with PCOS are positively associated with HOMA-IR. To explore the association between APOC3 and HOMA-IR, the ELISA results were further analyzed. The analysis of this association demonstrated a strong positive association between APOC3 and HOMA-IR (Fig. 5).

\section{Discussion}

A number of methods are currently available for the clinical diagnosis of IR; however, there is no compatible method available for the accurate and effective diagnosis of the IR status in patients with PCOS. In the present study, HOMA-IR was used to evaluate the IR in patients with PCOS due to its simplicity and clinical applicability. However, the HOMA-IR level differs significantly between different populations, and between patients of different ethnicities and age groups. Thus far, there is no uniform standard method available for measuring the cut-off value of HOMA-IR worldwide. According to a Chinese diabetes prevention collaborative study, the cut-off value was 2.69 (22), while a clinical trial of patients with PCOS aged between 15 and 19 years demonstrated that the physical upper limits of HOMA-IR were 2.69 (23). Therefore, in the present study, the HOMA-IR value of 2.69 was taken as the critical value of IR in patients with PCOS.

In the present study, 4 differentially expressed proteins, namely afamin, serotransferrin, complement $\mathrm{C} 3$ and apolipoprotein C3, were distinguished by 2D-DIGE and MALDI-TOF-MS/MS analysis. Since there were 2 spots (the spots score is respectively 1,955 and 2,012, as illustrated in Table III) identified as APOC3, the credibility of APOC3 as the differentially expressed protein increased. Western blot analysis was further used to verify the differentially expressed proteins between the PCOS-IR and PCOS-NIR groups. The results indicated that APOC3 was upregulated in the PCOS-IR group. Multiple studies have suggested that APOC3 is closely related to IR (24-26) and another independent study demonstrated that IR was also positively associated with the production of APOC 3 protein. Based on these studies, it was thus hypothesized that APOC 3 could be used as an appropriate diagnostic biomarker for women with PCOS with IR.

APOC3 is a protein containing 79 amino acids, mainly located in chylomicrons, very low-density lipoprotein, LDL and HDL (27). APOC3 plays an important role in regulating lipid metabolism, inhibiting lipid lipoprotein lipase, hepatic lipase and reducing lacteal protein. Clinical studies have demonstrated that concentrations of APOC3 in the very low-density lipoprotein (VLDL) and LDL are higher in patients with myocardial infarction (28), and plasma APOC3 and apolipoprotein $\mathrm{B}(\mathrm{apoB})$ act as independent factors to predict coronary heart disease $(29,30)$. It is generally estimated that abnormal lipid metabolism, particularly high triglyceride lipoprotein metabolism, is the main factor leading to atherosclerosis, while APOC3 can replace lipoprotein lipase, which leads to reduced lipolysis $(31,32)$. In addition, the inflammatory responses caused by APOC 3 in vascular endothelial cells may further aggravate atherosclerosis (26). As 
Table III. The 4 differential proteins identified by 2D-DIGE and MALDI-TOF-MS/MS in the PCOS-IR and PCOS-NIR groups.

\begin{tabular}{|c|c|c|c|c|c|c|c|c|}
\hline \multirow[b]{2}{*}{ No. } & \multirow[b]{2}{*}{ Spot score (no.) } & \multirow[b]{2}{*}{ Protein } & \multirow[b]{2}{*}{ Accession no. } & \multicolumn{2}{|c|}{ Theoretical } & \multirow[b]{2}{*}{ Protein score } & \multirow[b]{2}{*}{ Protein $(\%)$} & \multirow[b]{2}{*}{ IR/NIR } \\
\hline & & & & Mass (Da) & PI & & & \\
\hline 1 & 871 & Afamin & P43652 & $70,962.7$ & 5.64 & 130 & 100 & 1.66 \\
\hline 2 & 975 & Serotransferrin & P02787 & $79,280.5$ & 6.81 & 213 & 100 & 1.53 \\
\hline 3 & 1,028 & Complement C3 & P01024 & $188,569.5$ & 6.02 & 72 & 99.88 & 1.76 \\
\hline 4 & 1,955 & Apolipoprotein C3 & P02656 & $10,845.5$ & 5.23 & 72 & 99.88 & 1.47 \\
\hline 5 & 2,012 & Apolipoprotein C3 & P02656 & $10,845.5$ & 5.23 & 72 & 99.88 & 2.14 \\
\hline
\end{tabular}

Compared to the PCOS-NIR group, afamin, serotransferrin, complement C3 and apolipoprotein C3 were upregulated in the PCOS-IR group. Spots 1955 and 2012 were both identified as apolipoprotein C3. PCOS-IR, polycystic ovary syndrome patients complicated by insulin resistance; PCOS-NIR, polycystic ovary syndrome patients without insulin resistance; 2D-DIGE, two-dimensional difference gel electrophoresis; MALDI-TOF-MS/MS, matrix assisted laser desorption ionization/time-of-flight MS; IR/NIR, the difference ratio of the protein spots in the PCOS-IR group and the PCOS-NIR group.

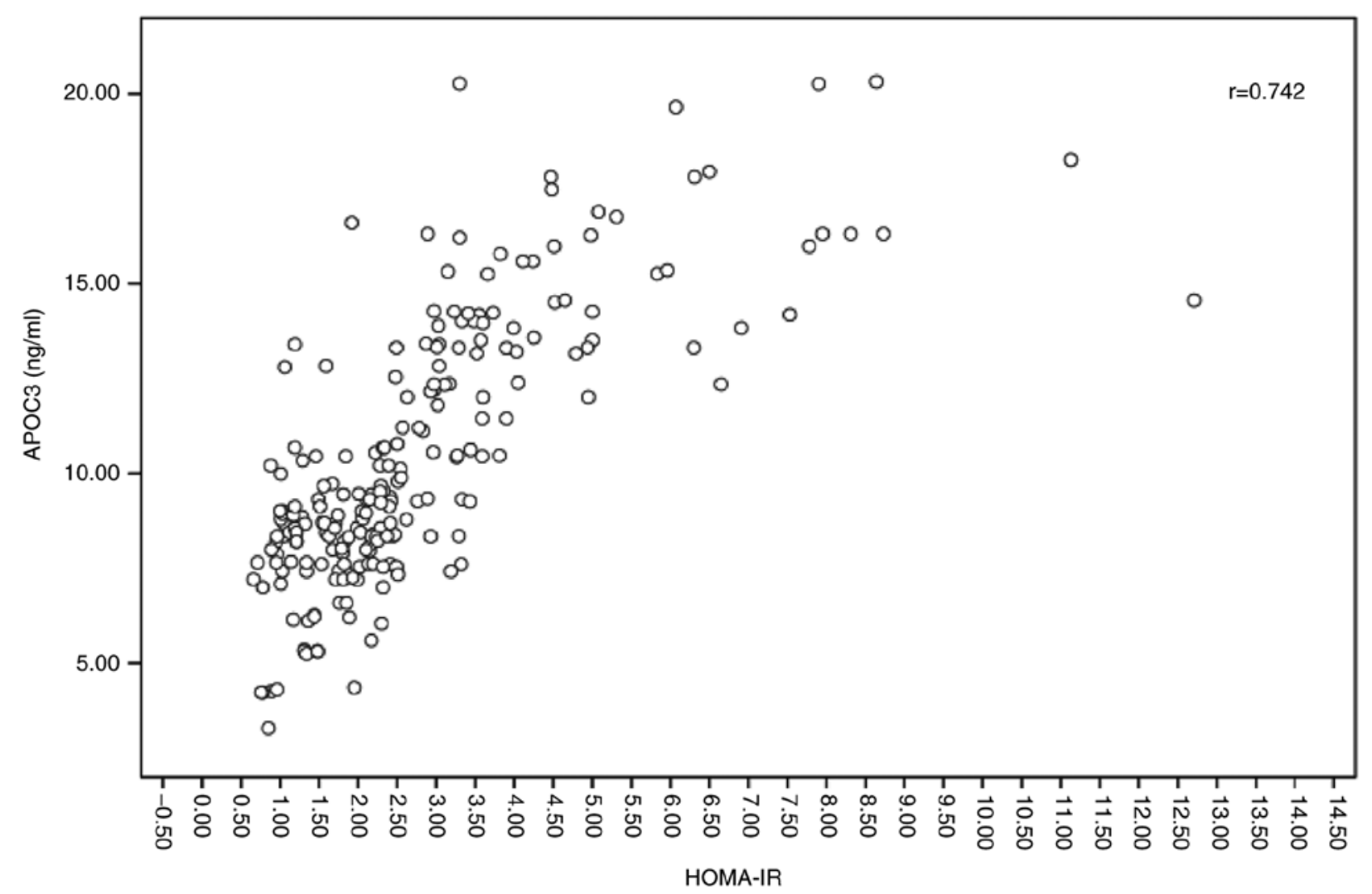

Figure 5. Association analysis between APOC3 and HOMA-IR. The ordinate represents the content of the target protein APOC3, and the abscissa represents the tolerance of glucose in PCOS-IR patients. APOC3, Apolipoprotein C3; HOMA-IR, homeostasis model assessment of insulin resistance.

is known, patients with PCOS also suffer from an increased risk of cardiovascular diseases and metabolic diseases. To the best of our knowledge, there is no evidence available to date to indicate that APOC3 may be used as a marker to predict the occurrence of long-term complications, such as diabetes, dyslipidemia and cardiovascular disease in women with PCOS. Therefore, further studies are warranted on this matter. In the present study, FPG and 3hBG levels in the PCOS-IR group were higher than those in the PCOS-NIR group, indicating a statistically significant difference $(\mathrm{P}<0.05)$. The results also revealed that the area under the ROC curve was measured at 0.936 (95\% CI, 0.901-0.972), the sensitivity was measured at $88.81 \%$, and the specificity was measured at $90.48 \%$. The present study also found that there was a positive association between APOC3 and HOMA-IR. Most importantly, it was demonstrated that APOC3 may be used as a biomarker of the IR status of patients with PCOS.

It is well known that PCOS is a lifelong disease, and a delay in the onset of the long-term complications associated with PCOS is considered highly beneficial to affected patients. The current study focused on the strong association of APOC3 with glucose homeostasis and lipid metabolism. 
However, further studies are required to determine whether the APOC3 gene can be used as a therapeutic target for IR in patients with PCOS. As the sample collection is still ongoing, in future studies, the authors aim to use other methods, such as ELISA and animal models to detect the therapeutic potential of APOC3 in patients with PCOS.

\section{Acknowledgements}

Not applicable.

\section{Funding}

The study was supported by the Science and Technology Planning Project of Guangdong Province (2014A020212237); the Key Project of Guangdong Provincial Administration of traditional Chinese Medicine: (20184005); the Science and Technology Planning Project of Guangdong Province (2017ZC0378); and the Guangzhou Municipal Science and Technology Innovation Council (201510010290).

\section{Availability of data and materials}

All data generated or analyzed during this study are included in this published article or are available from the first author on reasonable request.

\section{Authors' contributions}

LL and ZL designed the study. LL and JZh performed the experiments, participated in collecting the data and drafted the manuscript. JZe, BL and XP performed the statistical analysis and participated in its design. TL, JL, QT, XL, YY and ZC participated in the acquisition, analysis, or interpretation of data and drafted the manuscript. All authors read and approved the final manuscript.

\section{Ethics approval and consent to participate}

All patients signed written informed consent forms for participation, and this study was approved by the Ethics Committee of Guangdong Maternal and Child Health Hospital.

\section{Patient consent for publication}

Not applicable.

\section{Competing interests}

The authors declare that they have no competing interests.

\section{References}

1. Escobar-Morreale HF: Polycystic ovary syndrome: Definition, aetiology, diagnosis and treatment. Nat Rev Endocrinol 14: 270-284, 2018.

2. Wolf WM, Wattick RA, Kinkade ON and Olfert MD: Geographical prevalence of polycystic ovary syndrome as determined by region and race/ethnicity. Int J Env Res Pub He 15: E2589, 2018.

3. Sirmans SM and Pate KA: Epidemiology, diagnosis, and management of polycystic ovary syndrome. Clin Epidemiol 6: $1-13,2013$.
4. Legro RS, Arslanian SA, Ehrmann DA, Hoeger KM, Murad MH, Pasquali R and Welt CK; Endocrine Society: Diagnosis and treatment of polycystic ovary syndrome: An Endocrine Society clinical practice guideline. J Clin Endocrinol Metab 98: 4565-4592, 2013.

5. Azziz R, Marin C, Hoq L, Badamgarav E and Song P: Health care-related economic burden of the polycystic ovary syndrome during the reproductive life span. J Clin Endocrinol Metab 90: 4650-4658, 2005.

6. Burghen GA, Givens JR and Kitabchi AE: Correlation of hyperandrogenism with hyperinsulinism in polycystic ovarian disease. J Clin Endocrinol Metab 50: 113-116, 1980.

7. Dunaif A, Segal KR, Futterweit W and Dobrjansky A: Profound peripheral insulin resistance, independent of obesity, in polycystic ovary syndrome. Diabetes 38: 1165-1174, 1989.

8. Morales AJ,Laughlin GA, Butzow T, Maheshwari H, Baumann G and Yen SS: Insulin, somatotropic, and luteinizing hormone axes in lean and obese women with polycystic ovary syndrome: Common and distinct features. J Clin Endocrinol Metab 81: 2854-2864, 1996.

9. Cassar S, Misso ML, Hopkins WG, Shaw CS, Teede HJ and Stepto NK: Insulin resistance in polycystic ovary syndrome: A systematic review and meta-analysis of euglycaemic-hyperinsulinaemic clamp studies. Hum Reprod 31: 2619-2631, 2016.

10. Palomba S, Falbo A, Russo T, Rivoli L, Orio M, Cosco AG, Vero R, Capula C, Tolino A, Zullo F, et al: The risk of a persistent glucose metabolism impairment after gestational diabetes mellitus is increased in patients with polycystic ovary syndrome. Diabetes care 35: 861-867, 2012.

11. Zhang J, Li JM, Deng QS, Chen WF, Peng XH and Li L: Analysis of the metabolic characteristics of polycystic ovary syndrome under different states. Chin J Pract Gynecol Obstet 32: 446-449, 2016.

12. Rizzo M, Tyndall EK, Frontoni S, Jacoangeli F, Sarlo F, Panebianco F, Mistorni A, Di Renzo L, Calafiore R, Luca G and De Lorenzo A: Rapid and easy assessment of insulin resistance contributes to early detection of polycystic ovary syndrome. J Endocrinol Invest 36: 527-530, 2013.

13. Okamura Y, Saito F, Takaishi K, Motohara T, Honda R, Ohba T and Katabuchi H: Polycystic ovary syndrome: Early diagnosis and intervention are necessary for fertility preservation in young women with endometrial cancer under 35 years of age. Reprod Med Biol 16: 67-71, 2017.

14. Li X, Guo YR, Lin JF, Feng Y, Billig H and Shao R: Combination of Diane-35 and metformin to treat early endometrial carcinoma in PCOS women with insulin resistance. J Cancer 5: 173-181, 2014.

15. Tam CS, Xie W, Johnson WD, Cefalu WT, Redman LM and Ravussin E: Defining insulin resistance from hyperinsulinemic-euglycemic clamps. Diabetes Care 35: 1605-1610, 2012.

16. De Souza AL, Batista GA and Alegre SM: Assessment of insulin sensitivity by the hyperinsulinemic euglycemic clamp: Comparison with the spectral analysis of photoplethysmography. J Diabetes Complications 31: 128-133, 2017.

17. Kurl S,Zaccardi F, Onaemo VN,Jae SY, Kauhanen J, Ronkainen K and Laukkanen JA: Association between HOMA-IR, fasting insulin and fasting glucose with coronary heart disease mortality in nondiabetic men: A 20-year observational study. Acta Diabetol 52: 183-186, 2015.

18. Peplies J, Jiménez-Pavón D, Savva SC, Buck C, Günther K, Fraterman A, Russo P, Iacoviello L, Veidebaum T, Tornaritis M, et al: Percentiles of fasting serum insulin, glucose, HbAlc and HOMA-IR in pre-pubertal normal weight European children from the IDEFICS cohort. Int J Obesity (Lond) 38 (Suppl 2): S39-S47, 2014.

19. Mossmann M, Wainstein MV, Gonçalves SC, Wainstein RV, Gravina GL, Sangalli M, Veadrigo F, Matte R, Reich R, Costa FG and Bertoluci MC: HOMA-IR is associated with significant angiographic coronary artery disease in non-diabetic, non-obese individuals: A cross-sectional study. Diabetol Metab Syndr 7: $100,2015$.

20. Viswanathan S, Unlu M and Minden JS: Two-dimensional difference gel electrophoresis. Nat Protoc 1: 1351-1358, 2006.

21. McNamara LE, Dalby MJ, Riehle MO and Burchmore R: Fluorescence two-dimensional difference gel electrophoresis for biomaterial applications. J R Soc Interface 7 (Suppl 1): S107-S118, 2010.

22. Yin JH, Li M, Xu L, Wang Y, Cheng H, Zhao XY and Mi J: Insulin resistance determined by Homeostasis Model Assessment (HOMA) and associations with metabolic syndrome among Chinese children and teenagers. Diabetol Metab Syndr 5: 71, 2013. 
23. Li R, Yu G, Yang D, Li S, Lu S, Wu X, Wei Z, Song X, Wang X, Fu S and Qiao J: Prevalence and predictors of metabolic abnormalities in Chinese women with PCOS: A cross- sectional study. BMC Endocr Disord 14: 76, 2014.

24. Duivenvoorden I, Teusink B, Rensen PC, Romijn JA, Havekes LM and Voshol PJ: Apolipoprotein C3 deficiency results in diet-induced obesity and aggravated insulin resistance in mice. Diabetes 54: 664-671, 2005.

25. Lee HY, Birkenfeld AL, Jornayvaz FR, Jurczak MJ, Kanda S, Popov V, Frederick DW, Zhang D, Guigni B, Bharadwaj KG, et al: Apolipoprotein CIII overexpressing mice are predisposed to diet-induced hepatic steatosis and hepatic insulin resistance. Hepatology 54: 1650-1660, 2011.

26. Avall K, Ali Y, Leibiger IB, Leibiger B, Moede T, Paschen M, Dicker A, Daré E, Köhler M, Ilegems E, et al: Apolipoprotein CIII links islet insulin resistance to $\beta$-cell failure in diabetes. Proc Natl Acad Sci USA 112: E2611-E2619, 2015.

27. Ruiz-Narváez EA, Yang Y, Nakanishi Y, Kirchdorfer J and Campos H: APOC3/A5 haplotypes, lipid levels, and risk of myocardial infarction in the Central Valley of Costa Rica. J Lipid Res 46: 2605-2613, 2005.

28. Kavo AE, Rallidis LS, Sakellaropoulos GC, Lehr S, Hartwig S, Eckel J, Bozatzi PI, Anastasiou-Nana M, Tsikrika P and Kypreos KE: Qualitative characteristics of HDL in young patients of an acute myocardial infarction. Atherosclerosis 220 257-264, 2012.
29. Blankenhorn DH, Alaupovic P, Wickham E, Chin HP and Azen SP: Prediction of angiographic change in native human coronary arteries and aortocoronary bypass grafts. Lipid and nonlipid factors. Circulation 81: 470-476, 1990.

30. Sacks FM, Alaupovic P, Moye LA, Cole TG, Sussex B, Stampfer MJ, Pfeffer MA and Braunwald E: VLDL, apolipoproteins $\mathrm{B}, \mathrm{CIII}$, and $\mathrm{E}$, and risk of recurrent coronary events in the Cholesterol and Recurrent Events (CARE) trial. Circulation 102: 1886-1892, 2000.

31. Larsson M, Vorrsjö E, Talmud P, Lookene A and Olivecrona G: Apolipoproteins C-I and C-III inhibit lipoprotein lipase activity by displacement of the enzyme from lipid droplets. J Biol Chem 288: 33997-34008, 2013

32. Maeda N, Li H, Lee D, Oliver P, Quarfordt SH and Osada J: Targeted disruption of the apolipoprotein C-III gene in mice results in hypotriglyceridemia and protection from postprandial hypertriglyceridemia. J Biol Chem 269: 23610-23616, 1994.

(i) 9 This work is licensed under a Creative Commons Attribution-NonCommercial-NoDerivatives 4.0 International (CC BY-NC-ND 4.0) License. 